\title{
ICA and BP neural network based fingerprint recognition
}

\author{
Lu Zhao \\ Institute of Ideal Information Technology \\ Northeast Normal University \\ Changchun, China \\ Lulu727@gmail.com
}

\author{
Wenyong Wang \\ Institute of Ideal Information Technology \\ Northeast Normal University \\ Changchun, China \\ wenyongw@yeah.net
}

\begin{abstract}
According to the recognition of fuzzy fingerprint and the ones with strong noise, proposed a new method which combining the ICA (Independent Component Algorithm) and BP (Back Propagation) neural network. First, using the FastICA method to extract fingerprint characteristics, then classify and recognize them by a three- layers BP neural network. This method combines the local feature extraction capability of ICA, as well as the adaptive ability and robustness of BP neural network. Experiments show that this method has a higher recognition rate of the fingerprints with strong noise.
\end{abstract}

Keywords-Fingerprint recognition; strong noise; FastICA; BP neural network

\section{INTRODUCTION}

Fingerprint recognition, which has become to a reliable and common means of personal identification because of the uniqueness and conveniences of itself. In recent years, the research of fingerprint recognition has become to a research focus of the field of image processing and pattern recognition, and has been widely applied in banks, schools, public security and other all areas of society. Normally, fingerprint recognition can only recognize the fingerprints which are very clear, and in most cases, it can only recognize them individually. However, in some special circumstances, such as construction sites, factories and tourist areas, not only clean fingerprints cannot be guaranteed, but also a large number of fingerprints need to be identified. In these cases, traditional fingerprint recognition methods often appeared to be insufficient. At this time, ICA (Independent Component Algorithm) method [1] would play out its advantages.

ICA method bases on the higher-order statistical properties of signal. Therefore, the statistical independence of observed signal probability density function would be considered more comprehensively, the observed data would be linearly decomposed into statistics dependencies minimized components as well. In the aspect of classification and recognition, BP (Back Propagation) neural network has been widely applied by pattern Recognition System for its robustness. It is a nonlinear mapping from the input space to the output space. The classification would be realized by learning or changing the relationship between variables by adjusting their weights and the thresholds.

In this paper, a fingerprint recognition method based on the combination of ICA and BP neural network has been introduced. The fingerprint characteristics would be extracted by ICA method then be classified and recognized by a three- layers BP neural network.

\section{ICA-BASED FINGERPRINT FEATURE EXTRACTION}

ICA method is a new statistical signal processing method developed in 1990s. It has been widely applied in the research of fields such as communication speech signal processing, image processing and signal processing biological. The original purpose is to solve the blind signal separation problem. In the blind signal separation problem, the mixed mode of signals cannot be known, so statistical methods are the only kind of method to be adopted. It is assumed to have $n$-independent signal sources $s_{1}(t), \ldots, s_{m}(t)$, and $n$ - independent observables $x_{1}(t), \ldots, x_{\mathrm{n}}(t)$ [2].

Following is the relationship between the observed quantity and the source volume [3]:

$$
x(t)=A s(t)
$$

Where $x(t)=\left[x_{1}(t), \ldots, x_{n}(t)\right]^{T}, s(t)=\left[s_{1}(t), \ldots, s_{m}(t)\right]^{T}, A$ is a coefficient matrix , $n \times m$ dimensioned. Then it turns into another problem: known to the independence of $x(t)$ and $s$ $(t)$, finding out the estimation of $s(t)$.

Assuming the following formula:

$$
Y(t)=W x(t)
$$

Where $W$ is a coefficient matrix, $m \times n$ dimensioned. $y(t)$ is the estimation of $s(t)$, approaching $s(t)$ but not necessarily equal [4].

\section{A. Fingerprint ICA model}

Assuming each fingerprint image is linear mixed by $M$ source image, $S=\left[s_{1}, s_{2} \ldots s_{M}\right]^{T}, S \in R, M \times N$, which based on statistical independence. It means the actual fingerprint image matrix $X$ is linear mixed by source image matrix $S$ through mixing matrix $A$, in which $S$ is independent of each other. The aim is to approximately recovering $S$ by separation matrix $W$. which is given by (1).

It is assumed that $x_{i}$ is a fingerprint image, and a training set would be constructed by it. It is supposed that there are $m$ - fingerprint images and $n$ - pixels in each image. Each fingerprint image would spread in row, in order to be a onedimensional row vector, and connect them in row. Then an $m \times n$ dimension matrix has been constructed. This matrix is the $X$ in the random variable of ICA, its line represents a fingerprint image, and its row represents the pixels of the very image.

The separation matrix can be found out by FastICA algorithm, the output result would be: 


$$
\begin{gathered}
Y=W A S \\
W=A^{-1} \\
Y=S
\end{gathered}
$$

Where $Y=\left[y_{1}, y_{2}, \ldots, y_{\mathrm{M}}\right]^{T}, \quad Y \in R, m \times n$ demission, $\quad Y$ is an estimation of independent source image, its line represents an estimated source image.

\section{B. FastICA algorithm}

According to (3), (4) and (5) [5], the separation matrix $W$ would be find out, therefore the source image estimation matrix $Y$ would be find out as well. In this page, we adopt the FastICA algorithm, which has been introduced in [6], to find out the $W$. Following are the steps:

1) Demeaning the raw data $X$, then bleaching it, data $z$ could be get. The aim of bleaching is to get rid of the second-order correlation between observed signals, reduce dimensions of the data and simplify the subsequent process of extracting independent component[7].

2) Given $m$ to be the number of independent components that need to be extracted. Given $k=1$.

3) Initializing the weight vector ${ }_{w} \mathrm{k}(0)$, making \| ${ }_{w} \mathrm{k}(0) \mid 2=1$.

4) Adjusting the separation matrix $w$ :

$$
w_{k}(n)=E\left\{z g\left[w_{k}^{T}(n-1) z\right]\right\}-E\left\{g^{\prime}\left[w_{k}^{T}(n-1) z\right]\right\} \cdot w_{k}(n-1)
$$

5) Orthogonalizing the result:

$$
w_{k+1} \leftarrow w_{k+1}-\sum_{i=1}^{k} w_{k+1}^{T} w_{i} w_{i}
$$

6) Normalizing:

$$
w_{k+1} \leftarrow w_{k+1} / \sqrt{w_{k+1}^{T}+w_{k+1}}
$$

7) If ${ }_{w} \mathrm{k}$ and $-{ }_{w} \mathrm{k}$ are not pointing at the same direction, then $\mathrm{w}_{\mathrm{k}}$ is not converged, back to step 4).

8) If ${ }_{w} \mathrm{k}$ is converged, then find out an independent component $S_{k}=w_{k z}$.

9) Plus 1 to $k$, if $k \leq m$, then back to step 3), else the calculation is complete.

\section{BP NEURAL NETWORK}

Usually, a BP neural network has three layers: input layer, hidden layer and output layer, its topological structure is given by Figure 1:

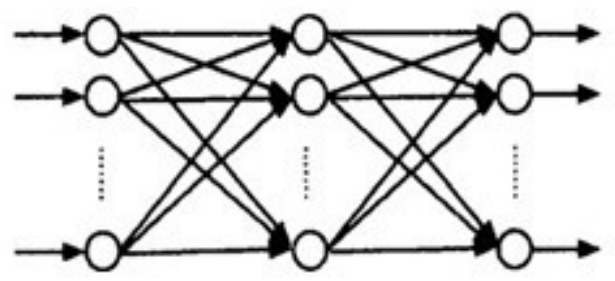

\section{Input layer Hidden layer Output layer}

Figure 1. Topological structure of BP neural network
The essence of a standard BP neural network is a gradient descent-based static optimization algorithm. Following are the steps to adjust its weights and thresholds:

1) Initializing the weights and thresholds of each neuron, inputting training samples and the desired output values.

2) Calculating output value of each layer from the input layer to the output layer.

3) Calculating Error signal of each layer, from output layer to the input layer.

4) Calculating the weight adjustment of each layer according to the error signa.

5) Adjusting the weight of each layer, calculating the error, if it is less than a certain threshold, then learning of one time is finished, else back to step 2).

\section{FINGERPRINT RECOGNITION OF THE METHOD COMBINES ICA WITH BP NEURAL NETWORK}

The overall structure diagram of the method combined ICA with BP neural network has been given by Figure 2 . Following are the implementation steps:

1) Preprocess of fingerprint image. The main job of this step is to scale the fingerprint image into a uniform size based on the principle of constant aspect ratio.

2) Extracting fingerprint characteristics of training set by ICA method. using the above fingerprint image training set $X=\left(x_{1}, x_{2}, \ldots, x_{m}\right)^{T}$. Extracting characteristics of this set.

3) Classification by BP neural network. Using threelayers BP neural network to classify the fingerprint characteristics extracted from the training set, and constantly adjusting errors.

4) Extracting fingerprint characteristics of test set by ICA method. Extracting fingerprint characteristics of test set using the method in step 2).

5) Recognition by BP neural network. Putting finger characteristics into the three-layered BP neural network to recognize them, then get the recognition result and calculate the recognition rate.

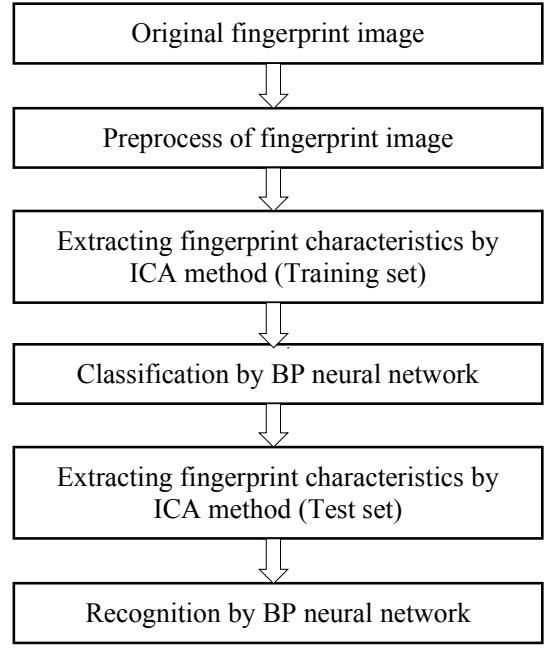

Figure 2. Structure diagram of ICA $-\mathrm{BP}$ algorithm 


\section{EXPERIMENTAL RESULTS AND ANALYSIS}

In order to verify the ability of the fingerprint separation under strong noise, as well as the characteristic extraction, in this experiment, random strong noise is mixed into fingerprint image, then separating them by FastICA algorithm.

From figure 3 , it can be find out that the fingerprint would be extracted from strong noise very well by FastICA algorithm. Belong to that, it can be seen that the extracted fingerprint is clear enough to be recognized.

During the recognition rate test, in this paper, a made fingerprint database has been used as experimental data. There are 50 people, each collected 10 times, totally 500 fingerprint images. Selecting 300 fingerprints randomly as training samples, the other 200 have been used as test set.

During this experiment, firstly noise has been added into fingerprints, then extracting their characteristics by ICA method. In order to compare the result, PCA method has also been used to extract the characteristics. Finally, BP neural network has been used to classify them. The BP neural network has been constructed by the method in [8]. The initial network weight has been selected from $(-1,1)$ randomly. Different hidden layer unit number has been selected to be trained and tested. The result has been shown in Table I. It can be seen that the recognition rate of ICA is higher than PCA. At the same time, the hidden layer unit number is not the more the better. It shows that in this experiment 50 is the best hidden layer unit number, under this circumstance, the recognition rate is higher and more recognition time saving.

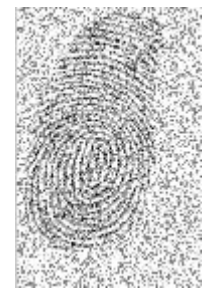

(a) Random mixed Image

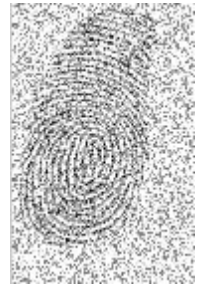

(b) Random mixed Image

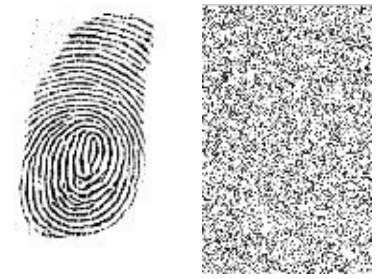

(c) Extracted Fingerprint and noise

Figure 3. Experiment results
TABLE I CLASSIFICATION RESULT

\begin{tabular}{|c|c|c|}
\hline Method & $\begin{array}{c}\text { Hidden layer } \\
\text { unit number }\end{array}$ & $\begin{array}{c}\text { Recognition } \\
\text { rate (\%) }\end{array}$ \\
\hline \multirow{3}{*}{ PCA } & 20 & 94.524 \\
\cline { 2 - 3 } & 50 & 96.499 \\
\cline { 2 - 3 } & 80 & 96.499 \\
\hline \multirow{2}{*}{ ICA } & 20 & 95.772 \\
\cline { 2 - 3 } & 50 & 96.255 \\
\cline { 2 - 3 } & 80 & 96.255 \\
\hline
\end{tabular}

\section{CONCLUSION}

In this paper, a fingerprint recognition method based on the combination of ICA and BP neural network has been introduced. Firstly extracting fingerprint characteristic by FastICA, then classifying them by BP neural network, and comparing the result by PCA. The experiment shows that ICA can get a higher recognition rate, especially when there is strong noise in them. ICA can separate them well and shows its advantage in characteristic extracting.

\section{ACKNOWLEDGMENT}

I gratefully acknowledge the valuable comments from Professor Wenyong Wang and my classmate Mengdi Wang who in particular called my attention to the research of fingerprint recognition.

\section{REFERENCES}

[1] Jie Wu and Zhengding Qiu, "Survey of feature extraction algorithms in palmprint recognition," Journal of Beijing Electronic Science and Technology Institute, vol. 13, February 2005, pp. 86-92.

[2] Jones MC and Sibson R, "What is Projection Pursuit," Journal of the Royal Statistical Society, vol. 150, 1978, pp. 1-36.

[3] Guibin Jin, Shuzhao Wang, Dezhi Niu and Zhuo Wang., "Fingerprint Blind Source Selection Based on FastICA in the Condition of Faintness and High Noise," Modern Electronics Technique, vol. 16, 2009, pp. 126-128.

[4] Ming Xiang, Xiaopei Wu and Mingsheng Liu, "Secondary Fingerprint Classification Algorithm Based on Independent Component Analysis," Computer Engineering, vol. 5, 2010, pp. 1618.

[5] Zhi Chen and Linlin Huang., "Recognition based on ICA and BP neural network." Journal of Beijing University of Aeronautics and Astronautics, vol. 34, March 2008, pp. 290-294.

[6] Aapo Hyvarinen, "Fast and Robust Fixedpoint Algorithms for Independent Component Analysis," IEEE Trans. On Neural Networks, vol. 10, March 1999, pp. 626-634.

[7] Aapo Hyvarinen, Karhunen J and Oja E, "Independent Component Analysis," New York: Wiley, 2001.

[8] Ke Han, Xiuchang Zhu and Huiyuan Wang, "Face recognition based on ICA and BP neural network," Information Technology and Informatization, vol. 2, 2006, pp. 50-52. 